\title{
Multicasting in Wireless Ad Hoc Networks Using ODMRP
}

\author{
Jayaprakash.S ${ }^{1}$ Ilamathi. $V^{2}$ Nithyakalyani. $\mathrm{K}^{3}$ \\ ${ }^{1}$ Asst.Professor,Department of MCA, Srinivasan Engineering College, Perambalur, Tamilnadu, (India), \\ ${ }^{2}$ Asst.Professor,Department of MCA, Srinivasan Engineering College, Perambalur, Tamilnadu, (India), \\ ${ }^{3}$ Asst.Professor,Department of MCA, Srinivasan Engineering College, Perambalur, Tamilnadu, (India).
}

\begin{abstract}
Wireless mesh networks are self-organized and usually without centralization control. Protocols in such networks are also required to be distributed for robustness and scalable. In this project localized mechanisms are adopted to support multicasting in wireless ad hoc networks without throttling unicast flows. The proposed scheme combines the layered multicast concept with the routing-based congestion avoidance idea to reduce the aggregated rate of multicast flows when they use excessive bandwidth on a wireless link. Our analysis and sweeping simulations show that the fully localized scheme proposed in this paper is effective in ensuring the fairness of bandwidth sharing between multicast and unicast flows in wireless ad hoc networks.
\end{abstract}

Keyword: wireless self configuring infrastructure less network, Mesh Topology, localized scheme, ODMRP.

\section{INTRODUCTION}

Wireless ad hoc networks such as mobile ad hoc networks and wireless mesh networks are selforganized and usually without centralised control. Protocols in such networks are also required to be distributed for robustness and scalable. If a distributed protocol only relies on local information and local actions for fulfilling its functional, then the protocol is also localised. In the sense of using only local resources, a localised protocol is usually efficient and scalablized, which are the basic characteristics required for protocols in wireless ad hoc networks. In this project localized mechanisms are adopted to support multicasting in wireless ad hoc networks without throttling unicast flows. Multicasting has emerged as one of the most focused areas in the field of networking. As the technologies and popularity of the Internet have grown, applications that require multicasting (e.g., video conferencing) are becoming more wides. Another interesting recent development has been the emergence of dynamically reconfigurable wireless ad hoc networks to interconnect mobile users for applications ranging from disaster recovery to distributed collaborative computing. Multicast plays a key role in ad hoc networks because of the notion of teams and the need to show data/images to hold conferences among them. Protocols used in static networks (e.g., DVMRP, MOSPF, CBT, and PIM), however, do not perform well in a dynamically changing ad hoc network environment. Multicast tree structures are fragile and must be readjusted continuously as connection to be changed. Furthermore, typical multicast trees usually require a global routing substructure such as link state or distance vector. The frequently exchange of routing vectors or link state tables, triggered by continuous topologies changed, yields excessive channel and processing overhead. Limited bandwidth, constrained power, and mobility of network hosts make the multicast protocol design particularly challenging. Multicast congestion control is source-based rate control, in which a multicast source regulates its transmission rate in response to loss indications (e.g. NAKs) from receivers. A number of specific source-based rate control schemes have been proposed, these represent important first solutions in a very large solution space. However, a number of basic issues remain open and have to be addressed by any source-based approach towards multicast congestion control.

\section{II. .PROBLEM STATEMENT AND CHALLENGES}

The scalability issue is essential to all multicast based protocols. A multicast congestion control protocol not only needs to scale to a large number of receivers but also needs to scale in a more heterogeneous environment with different link capacities and delays. Two resulting problems need to be addressed: feedback implosion and rate drop-to-zero.The implosion problem has been well explained in the literature on multicast error control, and various feedback suppression mechanisms have been introduced. However, all these mechanisms come with the cost of introducing extra delay in feedback. Feedback delay directly contributes to the responsiveness of congestion control schemes, the longer the delay the less the responsiveness. Additionally, in contrast to error control in which feedback is only triggered by packet losses discovered at receivers; in congestion control, the source needs constant feedback from the receivers to discover not only congestion but re-availability of resources as well. This continuous feedback should be well managed to avoid implosion and to a scalable can be achieve, yet they should also be delivered in a timely manner for sender to react to network 
congestion. The drop-to-zero problem is also known as loss multiplicity problem. The problem arises when receivers use packet losses as congestion signals and the source uses these signals to regulate its transmission rate without accumulated properly. When packets are lost multiple paths independent, receivers downstream of these paths will all send congestion signals to the source resulting in multiple rate drops at the source. In the current IP multicast model, the data source does not know the receiver topologies, hence cannot aggregate the congestion signals over receiver locations. Typically, when there are multiple bottleneck paths, the source has to adapt to the sum of the congestion signals generated on these paths and its rate will be quickly throttled as the number of congested paths increases.

\section{RELATED WORK}

ODMRP applies on-demand routing techniques to avoid channel overhead and improve scalability. It uses the concept at forwarding group, a set of nodes response for forwarding multicast data on shortest paths between any member pairs, to build a forwarding mesh topology for each multicast group. By maintaining and using a mesh topology related to the tree, the drawbacks of multicasting trees in mobile wireless networks (e.g. traffic concentration, frequent tree reconfigure, non-shortest path in a share the tree, etc.) are avoided. A softstate approach is taken in ODMRP to maintain multicast group members. No explicitly control message is required to leave the groups. We believe the reduction of storage overhead and the relaxed connectivity make ODMRP more scalable for large networks and more stable for mobile wireless networks. Instead of relying on end-to-end congestion control schemes, this project proposes a fully localized scheme in the network layer to support multicasting in wireless ad hoc networks while maintaining fairness with uncast flows. This integrates layered multicast with routing-based congestion avoidance to achieve its rate control over multicasting flow. The proposed scheme is fully localised. Each node acts based on local collected information and no additional interaction between nodes is required for the rate-control operations over multicast flows, except those required for standard multicast service. With the proposed scheme, a multicasting source encodes its signal into several layers of different priorities. The source then sends each layer to a separate multicast grouping. Receivers of the multicast source subscribe to these multicast groups and packets for all or some of these groups flow into the receivers can receives. At the same time, each intermediate node in the wireless ad hoc network monitoring its wireless links. When the link starts becoming congested, the node cuts the aggregate rate of multicast flows if the multicast flows are using excessive bandwidth on the links. The local rate-cut on multicasting flows is possible because each multicast flow has multiple layers, and layer priorities information is embedded in the multicast addresses of these layers. Our analysis and detailed frequently results show that the proposed scheme enables multicasting in wireless ad hoc networks and provides uncast flows their fair share of bandwidth.

\section{FUNCTIONAL DESIGN AND ANALYSIS}
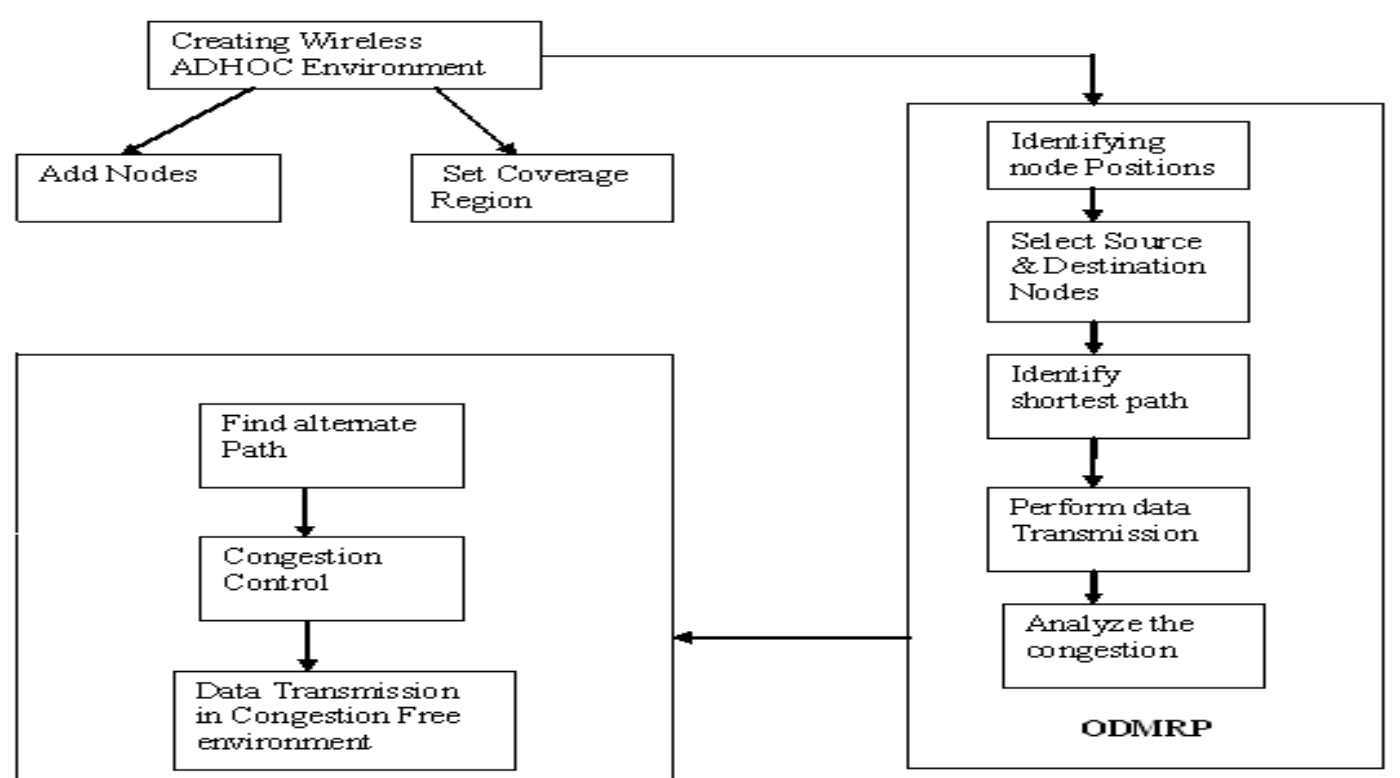

FULLY LOCALIZED CONTROL

Fig-1: Overview architecture 
Three set of functional steps needed as follows:

1. Creating wireless Adhoc Environment

2. On-Demand Multicast Routing Protocol (ODMRP)

3. Fully Localized Scheme

\section{Creating Wirless Adhoc Environment}

Wireless adhoc networks are self-organized and usually without centralized control. In this module we are going to create simulation of wireless adhoc environments.

The steps involved in this module are, measuring the number of nodes in every region. Fix the coverage region for each node. Design the adhoc environment that consists of nodes. Identify every node positions by getting the $\mathrm{X}$ and $\mathrm{Y}$ value positions of every node. The node can transmit data only up to the coverage region. If positions of the node exceed coverage regions the popup window displays the nodes are not in coverage distance

\section{Odmrp}

In this group membership and multicast routes are established and updated by the source on demand. In ODMRP, Identify the source node from where multicasting is going to be performed. Identify the destination nodes or group members that are going to receive the multicast data.

Design the routing table that consist of
i. Source node
ii Destination node
ii. Shortest path

Source node Destination node Shortest path Transmit the data according to the path present in the routing table. In this module heavy congestion will occur due to data transmission over same path at same time. Due to congestion in the network path the data may lose, this can be avoided by identifying the alternate path for every multicast data passing in the networks.

\section{Fully Localized Scheme}

There is no direct control over any uncast flows and assume that each uncast flow is controlled by the protocol without any queue management. Each node act based on locally collected information. Each node collects flow information about traffic traversing and congestion about the path. The fully localized control scheme identified the following Node positions Number of passing's per node

In this module we are going to implement layer priority information, lower layers have high priority and higher layers have less priority. The alternate route can be identified by the following methods. Based on the number of passings through the nodes the alternate flow is identified. If the routes are become more congested, we identify the alternate path based on the shortest distance to transmit data via other nodes. Finally the data transmission takes place between source to multiple destinations can be performed without any congestion

\section{EXPERIMENTAL RESULT}
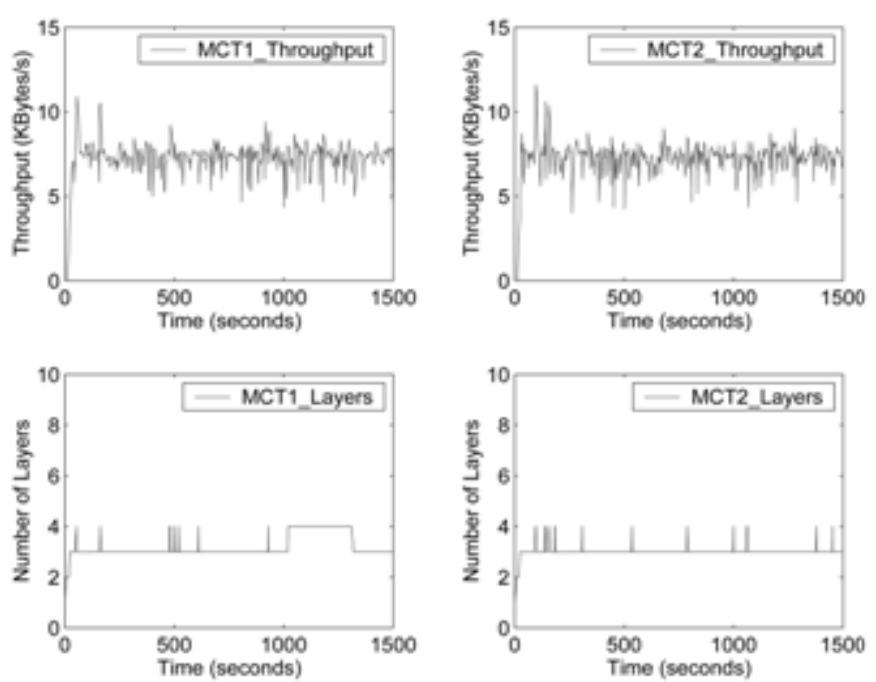

Fig-2: Individual multicast throughput and number of layers (RLM case) 

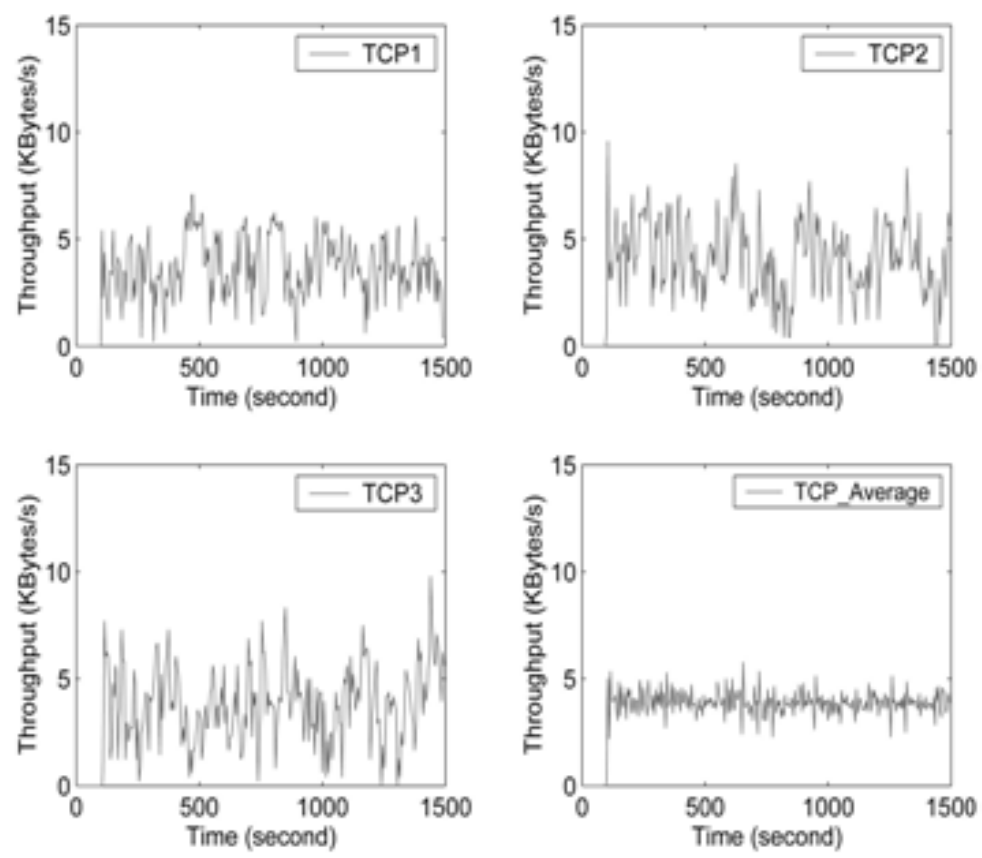

Fig-3: Individual TCP throughput and average per-flow TCP throughput .

\section{CONCLUSION}

Win runner is a automated functional GUI testing tool, as it allows us to record and playback the user actions from a vast variety of user applications as if a real user had manually executed those actions. It implements a automated Test Script Language (TSL) that allows customization and parameterization of user input. The test script is in the form of $\mathrm{C}$ language syntax, the user can edit script by adding some extra condition, to verify its functionality.

\section{ACKNOWLEDGMENTS}

This work was presented in part at the IEEE International Conferences on Communications (ICC), 2009. This work was can be taken in part of in our institution and support all staff members.

\section{REFERENCES}

[1]. Bhattacharyya.S, Towsley.D, and Kurose.J,( 1999), "The Loss Path Multiplicity Problem in Multicast Congestion Control," Proc. IEEEINFOCOM 99, pp. 856-863.

[2]. Byers.J, Luby.M, Mitzenmacher.M, and Rege.A (1998),"A Digital Fountain Approach to Reliable Distribution of Bulk Data," Proc. ACM SIGCOMM 98, pp. 56-67.

[3]. Byers.J, Luby. M, and Mitzenmacher. M (2001) "Fine-Grained Layer Multicasting," Proc. IEEE INFOCOM 01, Apr. 2001.

[4]. Gopalakrishnan.R , Griffioen.J, Hjalmtysson.G, Sreenan. C, \& Wen.S, " ( 2000) ,A Simple LoDifferentiation Approach to Layer Multicasting," Proc. IEEE INFOCOM 00.

[5]. Kwon.G and Byers.J, (2000) "Smooth Multi-rate Multicasting Congestion

[6]. Controls," Proc. IEEE INFOCOM '03. [6]Lee.S.-J, Gerla.M, and Chiang.C.-C,(1999), "On-Demand Multicast Routing Protocol," Proc. IEEE Wireless Comm. and Networking Conf. (WCNC '99), pp. 12981304.

[7]. McCanne.S, Jacobson.V, and Vetterli.M,(1996) " Receiver-Driven Layered Multicast," Proc. ACM SIGCOMM '96, pp. 117-130

[8]. Rhee.I, Balaguru.N, and Rouskas.G,(1999), "MTCP: Scalable TCPLike Congestion Control for Reliable Multicast," Proc. IEEE INFOCOM '99, pp. 1265-1273.

[9]. Rizzo.L, (2000)" PGMCC: A TCP-Friendly Single-Rate Multicast Congestion Control Scheme" Proc. ACM SIGCOMM '00.

[10]. Royer .E.M and Perkins. C.E,(Aug 1999), "Multicast Operation of the Ad Hoc On-Demand Distance Vector Routing Protocol," Proc. ACM MobiCom '99, pp. 207-218. 


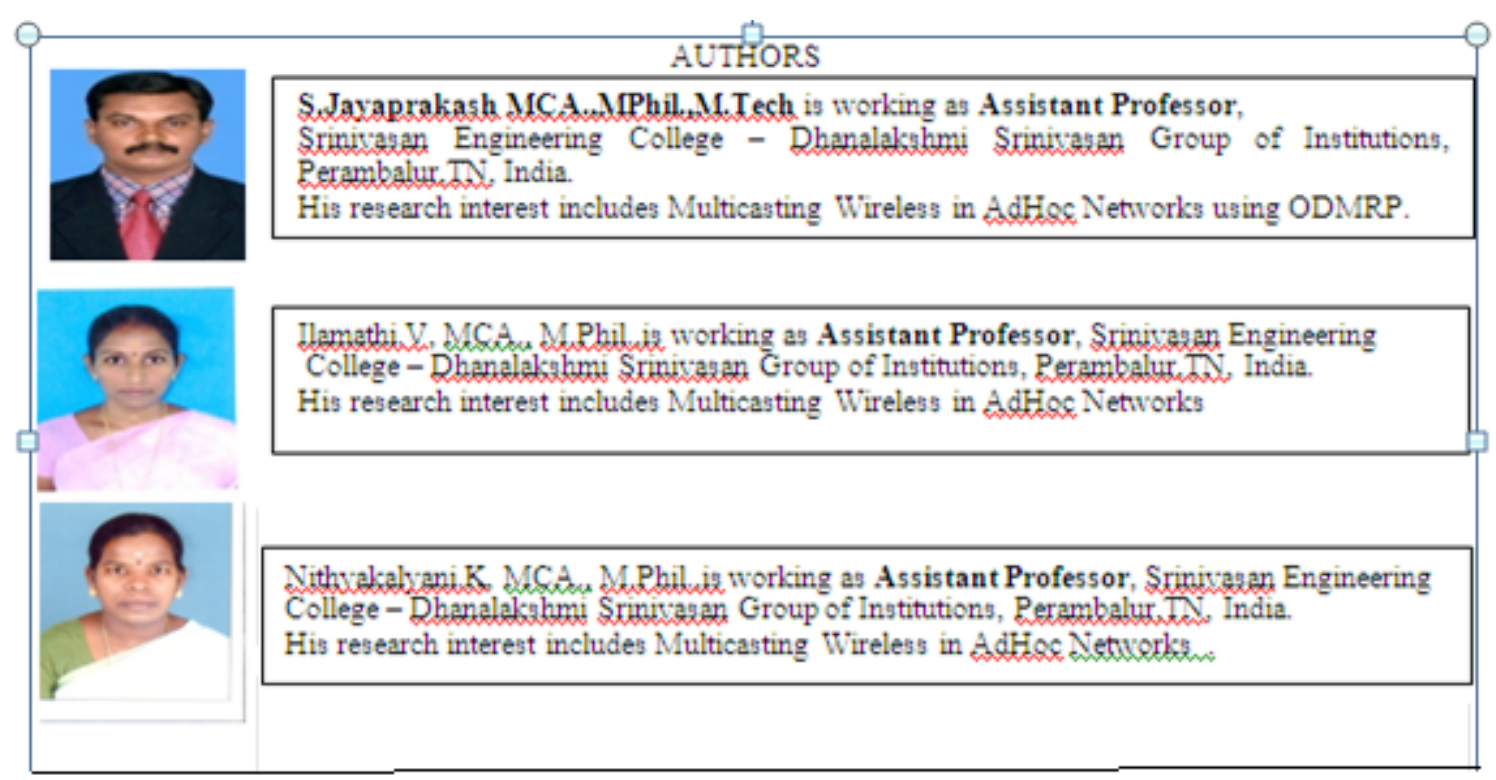

